\title{
Sammlung zur Repression - Zugang als demokratisches Recht
}

\author{
Die Nutzung der Stasi-Unterlagen der ehemaligen DDR
}

\section{Einleitung}

Wer einmal Gelegenheit hatte, in den Unterlagen des Ministeriums für Staatssicherheit der ehemaligen DDR (Stasi-Akten) zu lesen, dem erschiene es vermutlich nicht naheliegend, das Stasi-Unterlagen-Archiv im Rahmen einer Veranstaltungsreihe Literatur und Archiv zu thematisieren. Endlose Schachtelsätze, bürokratisch-militärische Wortungetüme und eine Fülle von Abkürzungen - die Sprache der Stasi (vom banalen Inhalt vieler Berichte ganz abgesehen) ist eher ein sprachliches Gegenbild zur Literatur.

Eine zeitgeschichtliche Assoziation zur Literatur wäre die Tatsache, dass kaum ein anderes Milieu von der Stasi so lückenlos überwacht und kontrolliert wurde wie die SchriftstellerInnen in der DDR. Wohl nirgends verfügte das Ministerium für Staatssicherheit (MfS) über ein so dichtes Spitzelnetz wie im sog. Sicherungsbereich Literatur. Der Schriftstellerverband war durchsetzt von Inoffiziellen Mitarbeitern (IM), missliebige KünstlerInnen waren Opfer zersetzender Maßnahmen. Kaum etwas fürchtete Stasi-Chef Erich Mielke so sehr wie die intellektuelle Unbotmäßigkeit.

Für den interdisziplinären Ansatz dieser Reihe, die „Logiken der Sammlung“ vergleichend zu beleuchten, dürfte das Stasi-Unterlagen-Archiv im Hinblick auf seine Besonderheiten allerdings nicht uninteressant sein. Nie zuvor sind die Hinterlassenschaften einer Geheimpolizei geschlossen übergeben und zeitnah zugänglich gemacht worden. Die Stasi hat niemals damit gerechnet, dass die Produkte ihrer streng konspirativen Tätigkeit eines Tages in diametraler Umkehr ihres Entstehungszwecks ausgerechnet auch den Opfern ihrer Maßnahmen gegenüber offengelegt werden würden.

Vor Abgabe nicht mehr benötigter Akten an Archive oder der Aufnahme in Sammlungen finden im Regelfall Prozesse der Anbietung, archivarischen Bewertung und Auswahl statt. Des Weiteren gelten für Unterlagen mit personenbezogenen Informationen normalerweise lange Schutzfristen, die einer Nutzung ohne Einwilligung grundsätzlich entgegenstehen. Die Stasi-Unterlagen wurden durch den plötzlich eintretenden politischen Umbruch der friedlichen Revolution und die Besetzungen der Stasi-Dienststellen 1989/90 quasi aus dem laufenden Betrieb 
,eingefroren'. Abgesehen von partiellen Aktenvernichtungen in der Schlussphase durch MfS-Mitarbeiter ${ }^{1}$ wurden die Hinterlassenschaften der Stasi weitgehend gesichert und geschlossen übernommen. Die Nutzbarmachung und zeitnahe Zurverfügungstellung dieses sensiblen und intransparent geordneten, geschlossenen Bestandes, verbunden mit einem Informationsauftrag, wurde zur besonderen Aufgabe der hierfür gebildeten Stasi-Unterlagen-Behörde unter Leitung eines vom Bundestag gewählten Beauftragten.

Das Verständnis für die Bedingungen der Nutzung von Stasi-Unterlagen und damit zusammenhängender Rechtsprobleme ist ohne Vergegenwärtigung der Umstände ihrer Entstehung wie auch ihrer Öffnung nicht möglich.

\section{Die Sammlung}

Das MfS war Geheimdienst, Ermittlungsorgan mit exekutiven Befugnissen und vor allem Geheimpolizei. Deren Aufgabe war es nach dem Motto Mielkes („Wir müssen alles wissen“), Informationen zu allem und jedem zu sammeln, der auch nur ansatzweise oder potenziell das Risiko darstellte, das herrschende Regime in Frage zu stellen. Mit einem gigantischen Überwachungsapparat von zuletzt 91.000 Hauptamtlichen und ca. 180.000 IM durchdrang die Stasi praktisch alle Gesellschaftsbereiche, um „feindlich-negative“ Kräfte aufzuspüren und zu bekämpfen (zum MfS allgemein vgl. Gieseke 2006; Gieseke 2007; Kowalzcuk 2013).

Hierin lag auch ein Grund, warum die Stasi, die ja ihrerseits nicht eigenständiger Akteur, sondern Herrschaftsinstrument der Sozialistischen Einheitspartei SED („Schild und Schwert der Partei“) war, für viele ein Symbol der Unfreiheit, Intransparenz und staatlichen Willkür darstellte, das in besonderer Weise das Gefühl von Ausgeliefertsein und Machtlosigkeit vermittelte. So geriet sie in besonderer Weise in den Fokus der friedlichen Revolution wie auch bei der späteren Aufarbeitung der SED-Diktatur.

Hauptwaffe des MfS war der IM (vgl. Müller-Enbergs 2001, 2008, 2010). ${ }^{2}$ Neben die IM-Berichte traten als Informationsquellen Observationen, Foto- und Videoüberwachungen, Postkontrollen und Abhörmaßnahmen auf (vgl. Kowalczuk 2016). ${ }^{3}$

1 Eine offizielle Aktenvernichtung fand mit Billigung des sog. Runden Tisches im Rahmen der beschlossenen Selbstauflösung der Hauptverwaltung A (Auslandsspionage) noch im Februar 1990 statt.

2 Zur Arbeit der IM vgl. die „Grundsatzdokumente“ auf der Website des BStU, v. a. die Richtlinie 1/76 des Ministeriums für Staatssicherheit (MfS 1976).

3 Zuständig für die DDR war die Abt. 26 des MfS. 
Gegen identifizierte Regimegegner wurden Maßnahmen eingeleitet, die von intransparenten Einflussnahmen auf Ausbildungs- und Arbeitsstellen wie auf Gerichtsverfahren (vgl. Fricke 1992, 253-273; Engelmann und Vollnhals 1999) bis zum Einsatz von Mitteln sog. Zersetzung, etwa durch Streuung falscher Gerüchte im persönlichen Umfeld oder Manipulationen in der Wohnung, zur psychischen Zermürbung reichten (vgl. Neubert 2003, 167, 185-186; Fuchs 1997; MfS 1976, 2.6). Diese Maßnahmen wurden in sog. operativen Vorgängen (OV) dokumentiert. Neben Opferakten gibt es Akten zu den IM, die neben den persönlichen Daten auch die gelieferten Berichte enthalten, Ermittlungsakten (sog. Untersuchungsvorgänge), eine umfangreiche Sammlung von NS-Akten, ${ }^{4}$ vom MfS verwahrte Justizakten, Personal („Kader“)-Akten sowie das allgemeine Verwaltungsschriftgut eines Ministeriums.

Die Organisation und Verwaltung der umfassend gesammelten personenbezogenen Informationen folgten den Zwecken und der Logik einer Geheimpolizei: Entscheidend war, auf diese möglichst schnell, effizient und umfassend zugreifen zu können. Kernstück der MfS-internen Archivabteilung (XII) waren die sog. zentralen Karteien, die dem Nachweis aller von den operativen Diensteinheiten des MfS erfassten Personen und angelegten Vorgänge dienten (vgl. Lucht 2015).

Die wichtigsten Findmittel waren - und sind bis heute - die sog. Personenkartei (F 16) und die sog. Vorgangskartei (F 22). In der Personenkartei sind alle Personen mit Geburtsort und Datum enthalten, die das MfS erfasst hat, ohne dass erkennbar ist, ob sie Betroffene, IM oder unbeteiligte Dritte waren. Mit 5,6 Mio. Karteikarten stellt sie für sich genommen schon ein beklemmendes Produkt des Überwachungsstaates dar. Die Vorgangskartei (1,2 Mio. Karten) gibt Aufschluss über die Art des Vorgangs, enthält aber nicht die Klarnamen der erfassten Personen und bezeichnet den IM nur mit seinem Decknamen. Informationen aus den getrennt voneinander untergebrachten Karteien waren - dem Prinzip der internen Konspiration folgend - nur im Einzelfall über die jeweils enthaltene Registriernummer einander zuzuordnen. Über die Vorgangskartei ist dann mit der entsprechenden Signatur der Zugriff auf die entsprechende Akte möglich.

Neben weiteren zentral geführten Karteien führten die zuletzt 44 Abteilungen des MfS sowie die 15 Bezirksverwaltungen und 209 Kreisdienststellen Tausende dezentraler Karteien. Insgesamt sind 41 Mio. Karteikarten überliefert (vgl. Lucht 2015, 181).

4 Diese wurden in der Hauptabteilung IX/11 des MfS verwaltet und dienten nicht in erster Linie strafrechtlichen Ermittlungen, sondern operativen und propagandistischen Zwecken gegenüber der Bundesrepublik. 
Die Archivabteilung des MfS war im Übrigen eher eine Altregistratur als ein Archiv, in dem die in den jeweils zuständigen Diensteinheiten angelegten Vorgänge nach einem eigenen - für Außenstehende intransparenten - System abgelegt, später aber durchaus auch verändert wurden. Insgesamt belief sich die 1989/90 übernommene Hinterlassenschaft des MfS auf mehr als 111 km Schriftgut, 41 Mio. Karteikarten, über 1,85 Mio. Fotodokumente, 2.865 Filme und Videos, 23.250 Tondokumente sowie 54 Datenprojekte des MfS. Hinzu kamen 15.500 Säcke mit zerrissenem Schriftgut.

\section{Sicherung, Öffnung und Erschließung}

Mit dem Stasi-Unterlagen-Gesetz (StUG) vom 20. Dezember 1991 wurde die gesetzliche Grundlage für die Öffnung und Nutzung der Unterlagen zu Zwecken der Diktaturaufarbeitung geschaffen. Damit war der Gesetzgeber einem in letzter Minute auf Druck der DDR-Bürgerrechtler dem Einigungsvertrag 1990 als Zusatz beigefügten Auftrag nachgekommen (vgl. Art. 1 der Zusatzvereinbarung zum Einigungsvertrag 1990). Dem waren kontroverse Diskussionen in Ost und West vorausgegangen (vgl. Schumann 1995). Die Bürgerrechtler forderten die Öffnung, wollten aber sicherstellen, dass die Betroffeneninformationen nicht ohne ihre Einwilligung durch Behörden weitergenutzt werden. Auch im Westen befürchteten Politiker, die Hinterlassenschaft dieses Spitzelapparats könnte für das wiedervereinigte Deutschland nur eine Belastung bis hin zu einer Spaltung der Gesellschaft mit sich bringen. Befürchtungen, die wie man heute weiß, unbegründet waren.

Die rechtspolitische Herausforderung, diese zum Teil höchst sensiblen und mit rechtsstaatswidrigen Mitteln erlangten Informationen, zudem ohne die archivrechtlich üblichen langen Sperrfristen, zeitnah für die Aufarbeitung zugänglich zu machen, hat das StUG durch folgende Eckpunkte gelöst:

- Ausschließliche Verwahrung, Verwaltung und Verwendung durch die Behörde des Bundesbeauftragten (vgl. $\S \S 1$ Abs. 2, 2 Abs. 1, §§ 7, 8, 9 StUG)

- Verwendung der Unterlagen nur für die und unter den Voraussetzungen der abschließend aufgeführten Zwecke (vgl. § 4 Abs. 1 StUG, sog. Verbot mit Erlaubnisvorbehalt)

- Strenger Schutz der Daten von Betroffenen und Dritten einerseits (vgl. $\S \S 1$ Abs. 1 Nr. 2, 3 Abs. 3, 4 Abs. 4, 5 Abs. 1, 11 Abs. 4 StUG). Nach $\S 5$ Abs. 1 StUG besteht ein grundsätzliches Verbot, Stasi-Unterlagen zum Nachteil von Betroffenen und Dritten zu verwenden) 
- Eingeschränkte Rechte für Personen, zu denen Unterlagen existieren, die als Unterlagen zu Mitarbeitern oder Begünstigten des MfS einzustufen sind (vgl. $\S \S 13$ Abs. 5, 32 Abs. 1 Nr. 3 StUG)

- Umfassende Schutzvorschriften zur Berücksichtigung schutzwürdiger Belange im Einzelfall (vgl. § 32 Abs. 3 Nr. 2 StUG)

Dies heißt konkret: Unterlagen mit Informationen zu Betroffenen oder Dritten können ohne deren vorherige Einwilligung nicht verwendet bzw. müssen anonymisiert werden. Ehemalige Mitarbeiter des MfS, Begünstigte oder deren Befehlsgeber müssen eine Herausgabe zu einem gesetzlich erlaubten Zweck auch ohne vorherige Einwilligung als Eingriff in ihr Recht auf informationelle Selbstbestimmung dulden. Für Personen der Zeitgeschichte und Amtsträger gelten bei der Verwendung durch Forschung und Medien gesonderte Regelungen (vgl. §§ 32 Abs. 1 Nr. 4, 32 Abs. 3 Nr. 3, 32a, 34 StUG). Höchstpersönliche Informationen sind in allen Fällen grundsätzlich von der Verwendung ausgeschlossen (vgl. § 32 Abs. 1 Satz 2, Abs. 3 Satz 2 StUG).

Zur juristischen Problemlösung, eine Verwendung der Stasi-Unterlagen unter verfassungskonformer Abwägung und dem Schutz der betroffenen Persönlichkeitsrechte zu ermöglichen, kam auf den Bundesbeauftragten die Herkulesaufgabe zu, in kürzester Zeit eine Behörde aufzubauen (vgl. Gauck 2003) ${ }^{5}$ und weitgehend ohne entsprechende Spezial- und Insiderkenntnisse die zum großen Teil ungeordneten Überlieferungen - bei gleichzeitig hohem Antragsaufkommen nutzbar zu machen. Eine Nutzung war zunächst überhaupt nur personenbezogen möglich. Von den 111 Aktenkilometern waren ca. die Hälfte bei der Übernahme nicht in den archivierten Ablagen, sondern in den Büros der MfS-Dienststellen in Bündeln sichergestellt worden. Sie mussten im Hinblick auf die künftige Nutzung für Aufarbeitungszwecke nicht nur personenbezogen, sondern nun auch sachthematisch erst erschlossen werden.

\section{Zugangsmöglichkeiten und Verwendungszwecke}

\section{a) Persönliche Akteneinsicht}

Mit dem Recht auf persönliche Akteneinsicht (vgl. §§ 12ff. StUG) wurde in weitreichender Weise das Postulat des „Right to Truth“ realisiert, das inzwischen im

5 Vgl. hierzu die Tätigkeitsberichte der BStU (inbes. BStU 1993, 6-7). Innerhalb von wenigen Jahren stieg die Mitarbeiterzahl von 60 auf über 3.000. Inzwischen hat die Behörde des BStU insgesamt ca. 1.400 Mitarbeiter an 14 Standorten (vgl. BStU 2019). 
Rahmen von Transitional-Justice-Prozessen international zum Begriff geworden ist (vgl. Quintana 1997, 10).

Opfer von Maßnahmen des MfS konnten nun Klarheit darüber erlangen, wie die Stasi in ihr Leben eingegriffen hatte (vgl. z. B. Fricke 1995), was das MfS über sie wusste und durch gesonderte Decknamenentschlüsselung auch, wer manchmal aus dem engsten persönlichen Umfeld - Informationen über sie geliefert hatte (vgl. § 13 Abs. 5 StUG). Ebenso wichtig für die Opfer der Stasi ist aber auch die Gewissheit, wer den Anwerbeversuchen widerstanden hatte.

Die Antragszahlen haben hier alle Erwartungen um ein Vielfaches übertroffen. ${ }^{6}$ Viele Menschen brauchen eine längere Zeit, bis sie sich zur Einsicht in die Stasi-Akten entschließen, wofür es verschiedene Gründe gibt. Der Umgang mit den Erkenntnissen liegt im persönlichen Bereich, lässt sich dementsprechend nicht allgemein erfassen. Der Staat kann durch die Möglichkeit der Akteneinsicht nur die Grundlage für eine persönliche Auseinandersetzung schaffen.

\section{b) Verwendung der Stasi-Unterlagen durch Gerichte und Behörden und weitere ersuchensberechtigte Stellen}

Für die strafrechtliche Aufarbeitung von Regimestraftaten (vgl. Marxen et al. 2007; Jahntz 2003, 309-335; § 23 StUG) waren die Stasi-Unterlagen wichtige, zum Teil entscheidende Beweismittel. Sie vollzog sich im Wesentlichen innerhalb von zehn Jahren nach der Wiedervereinigung. Am bekanntesten sind die sog. Mauerschützenprozesse (vgl. Rummler und Marxen 2002), bei denen der Bundesgerichtshof durch den wegweisenden Rückgriff auf die Menschenrechtspakte eine Bestrafung der Täter ermöglichte (vgl. BGHSt 39, 1-2, 168-169, 353-354; BGHSt 40, 218-219; Werle 2001, 3001-3008). Ersuchen von Ermittlungsbehörden gibt es vereinzelt auch noch gegenwärtig. ${ }^{7}$

Für die Aufhebung politisch motivierter Strafurteile (vgl. Engelmann und Vollnhals 1999; Fricke 2000) im Rahmen von gerichtlichen Rehabilitierungsverfahren bilden vornehmlich die beim MfS verwahrten Justizakten naturgemäß eine entscheidende Grundlage (vgl. §§ 20, 21 Abs. 1 Nr. 1 StUG i. V. m. dem Strafrechtlichen Rehabilitierungsgesetz).

Schwieriger gestalten sich behördliche Wiedergutmachungsverfahren, da die MfS-Akten in vielen Fällen nicht die vom Antragsteller erhofften Belege für beruf-

6 Noch im letzten Jahrzehnt lag die Zahl zum Teil über 100.000 p. a., im Jahr 2019 waren es noch 56.000 (vgl. BStU 2019, 30 und 84). Insgesamt betrug die Zahl der Personen, die eine persönliche Akteneinsicht beantragt haben, über 2,1 Mio.

7 Hierbei handelt es sich in der Regel um die Ermittlung in allgemeinen Mordfällen oder Terrorstraftaten (vgl. § 23 Abs. 1 StUG). 
liche Benachteiligungen oder Gesundheitsschäden infolge politischer Haft liefern (vgl. Lembcke 2009). ${ }^{8}$

Hinsichtlich der Überprüfung von Angehörigen des öffentlichen Dienstes, Abgeordneten von Parlamenten und kommunalen Körperschaften und weiteren wichtigen Funktionsträgern auf eine mögliche Stasi-Verstrickung ist im Gesetz detailliert geregelt, welche Stellen zu welchen Personen berechtigt sind, ein entsprechendes Überprüfungsersuchen zu stellen (vgl. §§ 20 Abs. 1 Nr. 1-6, 21 Abs. 1 Nr. 6-9 StUG). Das Gros der Überprüfungen vollzog sich bis zum Jahre 2006, seitdem ist nur noch ein enger begrenzter Personenkreis überprüfbar. Die Überprüfungsmöglichkeiten wurden 2006, 2011 sowie 2019 verlängert und bestehen nunmehr bis zum Jahr 2030 fort. Nach wie vor gibt es bei politischen Funktionen, auch im kommunalen Bereich, eine hohe Sensibilität gegenüber Stasi-Verstrickungen, wobei häufig der Umgang damit durch die betreffende Person ein Problem darstellt. Die Bewertung und die Entscheidung über Konsequenzen aus belastenden Mitteilungen trifft allein die ersuchende Stelle unter Berücksichtigung aller Umstände des Einzelfalles. ${ }^{9}$ So ist es im Bereich des Öffentlichen Dienstes in etwa der Hälfte dieser Fälle zu einer Entlassung der betreffenden Person gekommen. ${ }^{10}$ Im Ergebnis haben die Überprüfungen ohne Zweifel einen wichtigen Beitrag zum Aufbau einer rechtsstaatlichen Verwaltung in den neuen Bundesländern und zur Herstellung von Vertrauen in die öffentlichen Amts- und Funktionsträger nach der Wiedervereinigung geleistet, auch wenn die zum Teil einseitige Fokussierung auf die Stasi nicht immer angemessen gewesen war und ist.

\section{c) Forschung und Medien}

Mit dem Zugang für Forschung und Medien (vgl. §§ 32-34 StUG; BStU 2019, 38-39) wurden die Grundlagen für eine umfassende politische und historische Aufarbeitung der SED-Diktatur geschaffen, zu der die Forschung wie die Medien in den vergangenen Jahrzehnten entscheidend beigetragen haben. Die Verwendung personenbezogener Informationen stellt in diesem Bereich naturgemäß einen beson-

8 Vgl. hierzu die Tätigkeitsberichte der BStU sowie die Website der Union der Opferverbände kommunistischer Gewaltherrschaft e. V. (uokg.de). Durch die Einführung einer Opferrente für ehemalige politisch Inhaftierte im Jahre 2007 sind diese verfolgungsbedingten Nachteile gemildert worden.

9 Zur Überprüfung vgl. insbes. BStU 2007, 10-13; BStU 2019, 35-39.

10 Insoweit ist man auf Schätzungen angewiesen, da es auch wegen der Länderzuständigkeiten keine einheitliche Erfassung und Statistik gibt (vgl. Mothes und Schmidt 2002; Cevro-Institut 2017, 22-23). 
ders sensiblen Anwendungsbereich dar, da hier Persönlichkeitsrechte in besonderer Weise berührt und ggf. schutzbedürftig sind, zugleich aber mit dem Grundrecht der Presse- und Forschungsfreiheit und dem Aufarbeitungsinteresse in einem Spannungsverhältnis stehen.

Auch hier gilt die gesetzliche Unterscheidung zwischen Informationen $\mathrm{zu}$ Betroffenen und Dritten einerseits, zu denen grundsätzlich Einwilligungen beigebracht werden müssen, und Informationen zu Mitarbeitern andererseits (vgl. §§ 32 Abs. 1 Nr. 3 und 6, 34 StUG). Deren personenbezogenen Daten können unter Beachtung schutzbedürftiger Rechte herausgegeben werden, wenn sie in Bezug zu dem jeweiligen Aufarbeitungsvorhaben stehen. Personen der Zeitgeschichte, Amts- und Funktionsträger sind vor der Herausgabe sie betreffender Unterlagen zu benachrichtigen und haben die Möglichkeit, Einwände zu erheben, welche dann Gegenstand einer gerichtlich überprüfbaren Abwägung mit dem jeweiligen Aufarbeitungsinteresse seitens des BStU sind (vgl. §§ 32 Abs. 1 Nr. 4, 32a, 34 StUG). ${ }^{11}$ In der Regel kommt es zu gütlichen Einigungen mit den Beteiligten.

Die durch das StUG gestellten Anforderungen an die Verwendung personenbezogener Informationen binden den BStU als hoheitliche, der Grundrechtsbindung unmittelbar unterliegende Behörde bei der Herausgabe und zusätzlich auch den veröffentlichenden Antragsteller. Im Falle von Verstößen kann der Betroffene unter Umständen gegen beide gerichtlich vorgehen.

Unterlagen $\mathrm{zu}$ Betroffenen waren nach deren Tod der Verwendung dauerhaft entzogen. Im Jahre 2006 wurde im Interesse von Forschung und Medien eine an das Archivrecht angelehnte Schutzfrist von 30 Jahren zur Verwendung von Unterlagen mit personenbezogenen Informationen auch von Betroffenen nach deren Tod eingeführt (vgl. § 32 Abs. 1 Nr. 6 StUG, eingeführt durch das 7. Gesetz zur Änderung des Stasi-Unterlagen-Gesetzes; vgl. BGBl. 2006). In Ausnahmefällen ist eine Fristverkürzung auf zehn Jahre möglich, was aber restriktiv nur in Ausnahmefällen angewendet wird (etwa im Falle der Biografie zu einer verstorbenen Schriftstellerin mit gemischten Überlieferungen, zu der ansonsten nur IM-Unterlagen herausgabefähig wären).

\section{d) Grundlagenforschung und Unterrichtung der Öffentlichkeit}

Der BStU hat selbst einen gesetzlichen Aufarbeitungsauftrag (vgl. § 37 Abs. 1 Nr. 5 StUG). Sein Ziel ist es, die Öffentlichkeit über Struktur, Methoden und Wirkungs-

11 Diese differenzierende Regelung wurde im Jahre 2002 im Zusammenhang mit einem Rechtsstreit betr. beantragte Herausgaben zum ehem. Bundeskanzler Helmut Kohl eingeführt. Im Gegensatz zu manchen Befürchtungen hat sie sich bewährt und die Aufarbeitung nicht spürbar erschwert. 
weise des Staatssicherheitsdienstes zu unterrichten und hierbei die externe Forschung zu unterstützen.

Dies geschieht durch zahlreiche wissenschaftliche Publikationen zu grundsätzlichen Aspekten des MfS, Handbücher, Dauer- und Wanderausstellungen, Veranstaltungen, durch Bereitstellung von Materialien für die politische Bildung an Schulen sowie zunehmend auch über das Internet, seit 2015 gibt es eine Mediathek, die ständig erweitert wird.

Aktuelle laufende Forschungsvorhaben sind neben der Bestandsforschung etwa die Edition der zentralen Berichte des MfS an die Parteiführung (ZAIG) sowie die Mitarbeit am Projekt zur Provenienz-Forschung von Kulturgütern in der SBZ und DDR (vgl. BStU 2019, 11-12; BStU 2001, 46-47).

\section{Rechtsfragen der Nutzung}

\section{a) Differenzierung der Zugangsrechte nach Personenkategorien}

Schwierige Rechtsfragen ergeben sich im Einzelfall bei der für die Herausgabemodalitäten entscheidenden Kategorisierung von Unterlagen, wenn sich aus der Aktenüberlieferung nicht eindeutig feststellen lässt, ob es sich um Unterlagen zu einem IM handelt oder nicht, etwa wenn weder eine Verpflichtungserklärung noch IM-Berichte vorliegen. Entscheidend ist, ob sich die betreffende Person wissentlich und willentlich zur Informationsweitergabe bereit erklärt hat. Lässt sich dies bei lückenhafter Überlieferung nicht mit hinreichender Sicherheit aus den Unterlagen belegen, scheidet eine Herausgabe ohne Einwilligung aus. ${ }^{12}$

Die mit der im Zuge des Herausgabeverfahrens vorzunehmende Differenzierung der Unterlagen nach Personenkategorien verbundene Einschränkung des aus Art. 2, Abs. 1 i. V. m. Art. 1, Abs. 1 des Grundgesetzes abgeleiteten Rechts auf informationelle Selbstbestimmung für ehemalige Mitarbeiter des MfS (festgelegt in Art. 2, Abs. 1 und Art. 1, Abs. 1 des Grundgesetzes für die Bundesrepublik Deutschland), ist grundsätzlich durch das überwiegende Aufarbeitungsinteresse gerechtfertigt. Die Persönlichkeitsrechte der in den Unterlagen genannten ehemaligen IM dürften zwar zu Lebzeiten der betreffenden Personen mit zunehmendem Zeit-

12 Als politisch und juristisch besonders problematisch erwiesen sich die 2003 aus den USA zurückgeführten sog. Rosenholz-Dateien. Sie enthielten die mikroverfilmten Karteikarten und weitere Teilinformationen zur Auslandsspionage. Angesichts des Fehlens der vernichteten Akten reichten die vorhandenen Indizien in zahlreichen Fällen nicht aus, eine MfS-Mitarbeit im Sinne von $\S 6$ Abs. 4 Nr. 2 StUG anhand der Unterlagen schlüssig zu belegen und die Informationen ohne Einwilligung herauszugeben. 
ablauf tendenziell an Gewicht eher zunehmen. Mit der bloßen Herausgabe ist zwar noch kein Werturteil im konkreten Einzelfall verbunden, die formale Kategorisierung der „IM-Unterlage“ wird aber gleichwohl im praktischen Leben nicht selten bereits als eine Art Stigmatisierung empfunden. Insbesondere vor einer Veröffentlichung personenbezogener Informationen sollte daher stets durch den Autor geprüft werden, ob diese für den Aufarbeitungszweck erforderlich ist.

\section{b) Verwendung von Unterlagen zu Verstorbenen}

Fraglich ist, ob nach Ablauf der 30-Jahres-Frist nach Tod einer Person die Verwendung personenbezogener Unterlagen ohne Abwägung uneingeschränkt zulässig ist. Dies ist bei besonders sensiblen Unterlagen oder Informationen problematisch, insbesondere, wenn mit ihnen kein spezieller Erkenntniswert für die Aufarbeitung verbunden ist.

Eine politische Dimension hat die Frage, ob sog. menschenrechtswidrig erhobene Informationen 30 Jahre nach Tod einer Person der Zeitgeschichte oder eines Amtsträgers zugänglich gemacht werden dürfen. Nach der Rechtsprechung des Bundesverwaltungsgerichts sind menschenrechtswidrig erlangte Informationen auch dann von einer Verwendung ausgeschlossen, wenn sie die Amtsführung oder die zeitgeschichtliche Rolle einer Person betreffen. ${ }^{13}$ Dies betrifft vor allem wörtliche Abhörprotokolle, von denen allenfalls etwaige Zusammenfassungen des MfS ausschließlich Forschern zugänglich gemacht werden dürfen. Hier stellt sich die Frage, ob dieses Verwendungsverbot auch 30 Jahre nach Tod der Person fort gilt. Das wissenschaftliche oder mediale Aufarbeitungsinteresse (etwa an von der Stasi durch Abhörmaßnahmen erfasste Vorgänge in der Bundesrepublik) kollidiert hier mit der menschenrechtswidrigen Entstehung der Information. Der BStU hat bisher entsprechende Anträge abgelehnt. Hierfür spricht, dass die menschenrechtswidrige Entstehung einer Information nicht automatisch durch Zeitablauf „geheilt“ wird. Rechtspolitisch denkbar wäre hier unter Umständen die Einführung einer 60-Jahres-Frist.

\section{c) Nutzung von Stasi-Unterlagen im Zeitalter des Internets}

Aktuell stellt sich immer wieder die Frage, ob und in welchen Fällen das Einstellen von Stasi-Unterlagen ins Internet zulässig ist. Problematisch sind hierbei die Fälle, in denen sie personenbezogene Informationen enthalten.

13 Vgl. die Entscheidung des Bundesverwaltungsgerichts mit dem sog. 2. Kohl-Urteil vom 23. Juni 2004; kritisch dazu Heintschel von Heinegg (2004, 505-508); vgl. auch § 32 Abs. 1 Satz 3, Abs. 3 Satz 3 StUG. 
Publikationen, in denen zur Verfügung gestellte Stasi-Unterlagen nach §§ 32, 34 StUG verarbeitet wurden, können selbstverständlich auch über das Internet veröffentlicht werden. Auch können Personen die ihnen im Wege der persönlichen Akteneinsicht zugänglich gemachten Unterlagen in eigener Verantwortung publizieren. Die Frage ist aber, ob Stasi-Unterlagen mit personenbezogenen Informationen etwa zu IM als bloße Konvolute ins Netz gestellt werden dürfen.

Der Aktenzugang für Forscher und Medien nach $\S \S 32 \mathrm{ff}$. StUG erfolgt im Rahmen eines im Antrag konkret benannten Aufarbeitungsthemas. ${ }^{14}$ Gleiches gilt für die Herausgabe. Erhaltene Duplikate dürfen vom Empfänger weder für andere Zwecke verwendet oder an andere Stellen weitergegeben werden (vgl. § 33 Abs. 5 StUG; Zweckbindung und Weitergabeverbot). Diese rechtlichen Voraussetzungen der Verwendung können jedenfalls nach geltendem Recht nicht durch die technischen Möglichkeiten des Internets außer Kraft gesetzt werden.

\section{Weitere Herausforderungen}

Der Deutsche Bundestag hat beschlossen, dass die Aufarbeitung der SED-Diktatur fortgesetzt werden soll und der Gesamtbestand des Archivs wie auch die speziellen Zugangsregelungen des StUG zu den Stasi-Unterlagen erhalten bleiben (vgl. Deutscher Bundestag 2019a und 2019b). Dem Stasi-Unterlagen-Archiv stehen allerdings nach wie vor eine Reihe großer Herausforderungen bevor.

\section{a) Erschließung}

Die vollständige auch sachthematische Erschließung der Stasi-Unterlagen für die Bedürfnisse der Forschung hat sich als eine Generationenaufgabe erwiesen. Die Papier-Überlieferungen sind inzwischen ca. $\mathrm{zu}$ 97\% personenbezogen und $\mathrm{zu}$ $38 \%$ personen- und sachthematisch erschlossen. Bei Foto- und Tondokumenten ist dies zu 79\% bzw. 88\% der Fall (vgl. BStU 2019, 19).

Neben der Vervollständigung gilt es, vermehrt Findmittel auch extern und online zugänglich zu machen, was für verschiedene Sachaktenbestände schon geschehen ist. Insgesamt gibt es 320 Online-Findmittel des BStU, die sowohl in der Rechercheplattform (Argus) des Bundesarchivs als auch über das Archivportal Europa veröffentlicht wurden. Hier sind die Erschließungsangaben in

14 Dies ergibt sich nicht nur aus Gründen der Praktikabilität, sondern auch aus rechtlichen Gründen, denn anders wären der Aufarbeitungsbezug, die Abwägungen zur Erforderlichkeit der Herausgabe, etwa nach § 32a StUG sowie die Entscheidungen zur Einwilligung gar nicht möglich. 
einen transnationalen Recherchekontext eingebettet, etwa $\mathrm{zu}$ vergleichenden Fragestellungen zur Repressionsgeschichte in Mittel- und Osteuropa. Vermehrt sind Findmittel auch mit digitalisiertem Archivgut verlinkt, sodass etwa Tondokumente direkt abgehört werden können (vgl. BStU 2019, 24-25).

Es sollte ein Fernziel sein, ein einheitliches digitales Suchsystem für Sachund Personenrecherchen zu schaffen. Ein von der Fraunhofer-Gesellschaft eigens für den BStU entwickeltes Pilotverfahren zur virtuellen Rekonstruktion der zerrissenen Unterlagen soll nach einer Weiterentwicklung zur Qualitätsverbesserung zunächst in begrenztem Umfang fortgeführt werden (vgl. BStU 2019, 28-29).

\section{b) Digitalisierung}

Die Digitalisierung von Stasi-Unterlagen erfolgt zum einen aus Gründen des Bestandserhalts (vgl. BStU 2019, 27-28). Vorrangig musste es darum gehen, Tonund Videoaufzeichnungen, Fotoüberlieferungen, Mikrofilme und Mikrofiches vor der schleichenden Zerstörung zu bewahren.

Dem Bedürfnis der Nutzer - vornehmlich aus dem Bereich Medien und Forschung - entsprechend, wurde zum anderen die Möglichkeit geschaffen, antragsbezogen Stasi-Unterlagen in digitalisierter Form zur Verfügung zu stellen. Diese können inzwischen mit einer entsprechenden Software digital anonymisiert werden. Über ein beim BStU neu entwickeltes „Online-Abrufverfahren“ mittels eines Buttons auf der Website und eines separat übermittelten Codes können diese dann vom Antragsteller abgerufen werden. Auch können die Anträge online gestellt werden. Eine interne digitale Prozesskette ohne Medienbrüche wird es aber erst nach Einführung einer sog. e-Akte im Rahmen des „e-Government“ geben.

Eine Digitalisierung des gesamten Aktenbestandes der Stasi dürfte weder realistisch noch erforderlich sein, da viele Akten nur einmal oder wenige Male persönlich genutzt wurden und werden. Insofern wird man sich auf forschungsrelevante Vorgänge konzentrieren. Mit der Arbeit am Aufbau eines digitalen (Langzeit-)Archivs, von dem dann digitalisierte Akten für die verschiedenen Nutzungszwecke abgerufen werden können, wurde begonnen.

\section{c) Organisation und Struktur}

Nach dem Beschluss des Deutschen Bundestags soll das Stasi-Unterlagenarchiv bei fortbestehender Eigenständigkeit unter das Dach und in die Struktur des Bundesarchivs überführt werden (vgl. Deutscher Bundestag 2019a und 2019b). In diesem Zusammenhang sollen am Archivstandort Berlin-Lichtenberg perspektivisch unter anderen auch die DDR-Bestände des Bundesarchivs untergebracht werden. 
Synergieeffekte mit dem Bundesarchiv werden vor allem in archivfachlicher Hinsicht angestrebt (z. B. bei der Digitalisierung und Restaurierung). Auch soll eine gemeinsame Archivverwaltungssoftware entwickelt werden.

Eine inhaltlich sinnvolle Bündelung der Antrags- und Auskunftstätigkeit dürfte allerdings bis auf Weiteres wegen der unterschiedlichen Rechtsgrundlagen, Recherchen und Bearbeitungen auf Grenzen stoßen und ein Fernziel bleiben. Hinweise auf Bestände des Bundesarchivs werden den Antragstellern aber schon in der gegenwärtigen Praxis gegeben.

\section{Resümee}

Über sieben Millionen bearbeiteter Anträge bei einer sehr geringen Zahl von rechtlichen Auseinandersetzungen sowie das weitreichende internationale Interesse an den Erfahrungen mit der Aufarbeitung in Deutschland gerade auch im Hinblick auf die Stasi-Akten erlaubt es aus meiner Sicht, ungeachtet mancher Kritik, die Öffnung der Stasi-Akten als erfolgreichen Beitrag zur Überwindung der SED-Diktatur anzusehen. Auch wenn zuweilen die Gefahr einer einseitigen Fokussierung auf das MfS bestand, so war und ist die Brechung des Herrschaftswissens der Stasi ein entscheidendes Element zur Entlarvung und Demaskierung der Diktatur und damit zur Verhinderung von Legendenbildungen.

Auch wenn das wiedervereinigte Deutschland durch den Untergang des SEDRegimes, der Auflösung der Stasi und des gesamten Staates DDR günstigere Voraussetzungen für die Aufarbeitung des geschehenen Unrechts hatte als andere Länder, so war die Öffnung und Nutzung der Stasi-Akten gerade auch für ihre Opfer ein Impulsgeber für die Prozesse des Transitional Justice in vielen Ländern. ${ }^{15}$

Der Doppelcharakter des BStU als Datenschutzbehörde und als von Bürgerrechtlern erkämpfte Aufarbeitungsinstitution musste manche Spannungen mit sich bringen, extern wie intern, aber gerade auch diese führten in der Regel zu tragfähigen und weithin anerkannten Ergebnissen in der stetigen Balance zwischen rechtsstaatlich notwendiger Bürokratie und der Wiederherstellung von

15 In zahlreichen Ländern Osteuropas, Asiens, Afrikas und Lateinamerikas wurden Prozesse zur Aufarbeitung von Diktaturen, staatlicher Gewalt und Bürgerkriegen eingeleitet und entsprechende Gremien und Institutionen ins Leben gerufen. In diesem Umfang gab und gibt es vielfältige internationale Kontakte, Erfahrungsaustausche und Unterstützung durch international tätige Organisationen (vgl. United Nations 2015; BStU 2010; Cevro-Institut 2017; Werle und Vormbaum 2018). 
Transparenz und Vertrauen für die betroffenen Menschen. Die Stasi-Unterlagen werden nach den aktuellen Beschlüssen des Deutschen Bundestages in dem bisherigen Umfang ohne generelle Befristung dauerhaft zugänglich bleiben.

\section{Literaturverzeichnis}

BStU. 1. Tätigkeitsbericht des Bundesbeauftragten des Staatssicherheitsdienstes der ehemaligen Deutschen Demokratischen Republik 1993. Berlin, 1993. https://www.bstu.de/ assets/bstu/de/Downloads/bstu_01-taetigkeitsbericht_1993.pdf (15.12.2019).

BStU. 5. Tätigkeitsbericht der Bundesbeauftragten für die Unterlagen des Staatssicherheitsdienstes der ehemaligen Deutschen Demokratischen Republik. Berlin, 2001. https://www. bstu.de/assets/bstu/de/Downloads/bstu_05-taetigkeitsbericht_2001.pdf (15.12.2019)

BStU. 7. Tätigkeitsbericht des Bundesbeauftragten des Staatssicherheitsdienstes der ehemaligen Deutschen Demokratischen Republik 2005. Berlin, 2007. https://www.bstu. de/assets/bstu/de/Downloads/bstu_07-taetigkeitsbericht_2005.pdf (15.12.2019).

BStU. 14. Tätigkeitsbericht des Bundesbeauftragten des Staatssicherheitsdienstes der ehemaligen Deutschen Demokratischen Republik für die Jahre 2017 und 2018. Berlin, 2019. https://www.bstu.de/assets/bstu/de/Publikationen/TB_14_barrierearm.pdf (15.12.2019).

BStU. Das „Europäische Netzwerk der für die Geheimpolizeiakten zuständigen Behörden“. Ein Reader zu ihren gesetzlichen Grundlagen, Strukturen und Aufgaben. Berlin, 2010. https:// www.bstu.de/assets/bstu/de/Downloads/international_reader-europaeisches-netzwerk_ deutsch.pdf (15.12.2019).

Bundesgesetzblatt (BGBI.). Siebtes Gesetz zur Änderung des Stasi-UnterlagenGesetzes vom 21.12.2006. 3326-3331. http://www.bgbl.de/xaver/bgbl/start. xav?startbk=Bundesanzeiger_BGBI\&jumpTo=bgbl106s3326.pdf (15.12.2019).

Cevro-Institut (Hg.). Memories of Nations, Democratic Transitional Guide. 2017. www.cevro.cz/ guide (15.12.2019).

Deutscher Bundestag. Antrag der Fraktionen der CDU/CSU und SPD. Die Aufarbeitung der SED-Diktatur konsequent fortführen. Drucksache 18/8705. 7.6.2019a. https://www.bstu. de/assets/bstu/de/Downloads/bstu-in-zukunft_bundestagsdrucksache_18-8705.pdf (15.12.2019).

Deutscher Bundestag. Gesetzentwurf der Bundesregierung. Entwurf eines Neunten Gesetzes zur Änderung des Stasi-Unterlagen-Gesetzes. BT-Ds 19/11329. 26.9.2019b. http://dipbt. bundestag.de/dip21/btd/19/113/1911329.pdf (15.12.2019).

Einigungsvertrag. Vereinbarung zwischen der Deutschen Demokratischen Republik und der Bundesrepublik Deutschland zur Durchführung und Auslegung des am 21. August 1990 in Berlin unterzeichneten Vertrages zwischen der Bundesrepublik Deutschland und der Deutschen Demokratischen Republik über die Herstellung der Einheit Deutschlands. http://www.verfassungen.de/ddr/einigungsvertrag90-i.htm (15.12.2019).

Engelmann, Roger. „Der Weg zum Stasi-Unterlagen-Gesetz“. Stasi-Akten zwischen Politik und Zeitgeschichte. Eine Zwischenbilanz. Hg. Siegfried Suckut und Jürgen Weber. München: Olzog, 2003. 81-100.

Engelmann, Roger, und Clemens Vollnhals (Hg.). Justiz im Dienste der Parteiherrschaft, Rechtspraxis und Staatssicherheit. Berlin: Links, 1999. 
BGHSt 39, 1. Entscheidungen des Bundesgerichtshofes in Strafsachen. Mauerschützen I.

3.11.1992. http://www.servat.unibe.ch/dfr/bs039195.html (15.12.2019).

BGHSt 40, 218. Entscheidungen des Bundesgerichtshofes in Strafsachen. Mittelbare Täterschaft hoher DDR-Funktionäre. 26.7.1994. http://www.servat.unibe.ch/dfr/bs040218.html (15.12.2019).

Fricke, Karl-Wilhelm. Politik und Justiz in der DDR. Zur Geschichte der politischen Verfolgung 1945-1968. Köln: Verl. Wiss. u. Politik, 1992.

Fricke, Karl-Wilhelm. Akten-Einsicht, Rekonstruktion einer politischen Verfolgung. Berlin: Links, 1995.

Fricke, Karl-Wilhelm. Der Wahrheit verpflichtet, Texte aus fünfJahrzehnten zur Geschichte der DDR. Berlin: Links, 2000.

Fuchs, Jürgen. Unter Nutzung der Angst - Die „leise“ Form des Terrors, Zersetzungsmaßnahmen des MfS. Berlin: BStU 1997. URN: urn:nbn:de:0292-97839465720848.

Gauck, Joachim. „Rückblick auf die Anfänge der Behörde des Bundesbeauftragten“. Stasi-Akten zwischen Politik und Zeitgeschichte. Eine Zwischenbilanz. Hg. Siegfried Suckut und Jürgen Weber. München: Olzog, 2003. 17-26.

Gieseke, Jens. Der Mielke-Konzern. Die Geschichte der Stasi 1945-1990. 2. Aufl. München: Dt. Verl.-Anst., 2006

Gieseke, Jens (Hg.). Staatssicherheit und Gesellschaft. Studien zum Herrschaftsalltag in der DDR. Göttingen: Vandenhoeck \& Ruprecht, 2007.

Heintschel von Heinegg, Wolff. „Herausgabe und Verwendung von Stasi-Unterlagen mit personenbezogenen Informationen an die Presse. Anmerkungen zum Urteil des BVerwG vom 23. Juni 2004 (Fall Kohl)“. AfP Zeitschrift für Medien- und Kommunikationsrecht 6 (2004): 505-508.

Jahntz, Bernhard. „Die juristische Aufarbeitung der SED-Herrschaft. Eine vorläufige Bilanz“. Stasi-Akten zwischen Politik und Zeitgeschichte. Eine Zwischenbilanz. Hg. Siegfried Suckut und Jürgen Weber. München: Olzog, 2003. 309-335.

Kowalzcuk, Ilko-Sascha. Stasi konkret. Überwachung und Repression in der DDR. München: Beck, 2013.

Kowalzcuk, Ilko-Sascha. „Aktenlagen, Zum Mit- und Abhören im geteilten Deutschland“. „AktenEinsichten “. Beiträge zum historischen Ort der Staatssicherheit. Hg. Helge Heidemeyer. Berlin: BStU, Abt. Bildung und Forschung, 2016. 13-23. URN: urn:nbn:de:0292978394213077909.

Lembcke, Oliver W. „Rehabilitierung politisch Verfolgter in der DDR, Politisches Programm und Praxis des Rechts“. Politisches Denken. Jahrbuch 2009. Hg. Volker Gerhardt, Rinhard Mehring, Henning Ottmann, Martyn P. Thompson und Barbara Zehnpfennig. Berlin: Duncker \& Humblot, 2009. 167-196.

Lucht, Roland. „Karteien, Speicher, Datenbanken“. Das Gedächtnis der Staatssicherheit. Die Kartei- und Archivabteilung des MfS. Hg. Karsten Jedlitschka, Karsten und Philipp Springer. Göttingen: Vandenhoeck \& Ruprecht, 2015. 167-198.

Marxen, Klaus, Gerhard Werle, und Petra Schäfter. Strafverfolgung von DDR-Unrecht. Fakten und Zahlen. Berlin: Stiftung zur Aufarbeitung der SED-Diktatur, 2007.

Müller-Enbergs, Helmut (Hg.). Inoffizielle Mitarbeiter des Ministeriums für Staatssicherheit. Tl. 1-3, Berlin: Links, 2001, 2008, 2010.

MfS. Richtlinie 1/76 zur Entwicklung und Bearbeitung Operativer Vorgänge (OV), Pkt. 2.6. https://www.bstu.de/assets/bstu/content_migration/DE/Wissen/MfS-Dokumente/ Downloads/Grundsatzdokumente/richtlinie-1-76_ov.pdf (15.12.2019). 
Mothes, Jörn, und Jochen Schmidt. „Die Aufarbeitung der Vergangenheit“. Deutschland OstDeutschland West. Eine Bilanz. Hg. Hans-Georg Wehling. Opladen: Leske + Budrich, 2002. 54-69.

Neubert, Erhart. „Oppositionelle im Visier der Stasi“. Stasi-Akten zwischen Politik und Zeitgeschichte. Eine Zwischenbilanz. Hg. Siegfried Suckut und Jürgen Weber. München: Olzog, 2003. 167-197.

Rummler, Toralf, und Klaus Marxen (Hg.). Strafjustiz und DDR-Unrecht. Dokumentation. Bd. 2. Gewalttaten an der deutsch-deutschen Grenze. Teilbd. 1. Berlin, New York: de Gruyter, 2002.

Schumann, Silke. Vernichten oder offenlegen? Zur Entstehung des Stasi-Unterlagen-Gesetzes. Berlin: BStU, 1995.

StUG. Gesetz über die Unterlagen des Staatssicherheitsdienstes der ehemaligen Deutschen Demokratischen Republik (Stasi-Unterlagen-Gesetz - StUG). Berlin, 2018. https://www. bstu.de/assets/bstu/de/Downloads/stasi-unterlagen-gesetz-StUG.pdf (15.12.2019).

United Nations. UN-Report of the Special Rapporteur on the Promotion of Truth, Justice, Reparation and Guarantees of Non-Recurrance Pablo de Greiff, UN Human Rights Council A/HRC/30/42. 2015. https://www.ohchr.org/Documents/Issues/Truth/A-HRC-30-42.pdf (15.12.2019).

Quintana, Antonio Gonzales: UNESCO-Report, Archives of the Security Services of Former Repressive Regimes, Report prepared for the UNESCO on behalf of the International Council of Archives. Paris, 1997.

Werle, Gerhard, und Gerhard Vormbaum. Transitional Justice. Vergangenheitsbewältigung durch Recht. Berlin, Heidelberg: Springer, 2018.

Werle, Gerhard. „Rückwirkungsverbot und Staatskriminalität“. Neue Juristische Wochenschrift 54.41 (2001): 3001-3008. 